\section{Impacto das Unidades Básicas de Saúde na duração do aleitamento materno exclusivo}

\section{Impact of primary health care units' practice on the duration of exclusive breastfeeding}

\section{Maria Inês Couto de Oliveira}

Coordenadora do Grupo Técnico Interinstitucional de Incentivo ao Aleitamento Materno

Programa de Assistência Integral à Saúde da Mulher, Criança e Adolescente

Secretaria de Estado de Saúde do Rio de Janeiro

Rua México $128 / 4^{\circ}$ andar, Centro

20031-142. Rio de Janeiro, RJ - Brasil

Icamacho@ensp.fiocruz.br

\section{Luiz Antonio Bastos Camacho}

Escola Nacional de Saúde Pública

Fundação Oswaldo Cruz

Pesquisador do Departamento de Epidemiologia

\section{Resumo}

A Organização Mundial de Saúde recomenda o aleitamento materno exclusivo nos primeiros 6 meses de vida. Foi realizado um estudo para verificar o impacto de um conjunto de procedimentos e estratégias efetivas de promoção, proteção e apoio à amamentação, realizadas no pré-natal e na pediatria de unidades básicas de saúde, sobre a amamentação exclusiva em bebês menores de 6 meses. Avaliou-se práticas como a realização de grupos de apoio à amamentação e visitas domiciliares, a informação sobre a importância do início precoce da amamentação, da livre demanda e sobre os riscos do uso de mamadeiras e chupetas, a orientação quanto à pega, posição, ordenha e contracepção, e o apoio emocional. Este conjunto de estratégias e procedimentos foi selecionado com base em uma revisão sistemática e gerou uma proposta de "Dez Passos para o Sucesso da Amamentação na Atenção Básica à Saúde" e uma metodologia de avaliação elaborada nos moldes da Iniciativa Hospital Amigo da Criança. Essa metodologia foi aplicada na avaliação de 24 unidades básicas de saúde do Estado do Rio de Janeiro. O desempenho foi considerado regular em 13 unidades e fraco em 11 unidades. Foi encontrada uma expectativa de duração do aleitamento materno exclusivo de 1,6 meses para os bebês menores de 6 meses assistidos pelo bloco de unidades de desempenho regular, e de 1,1 mês para os bebês acompanhados pelo bloco de desempenho fraco. Não foram encontradas diferenças significativas entre as características sociodemográficas das mulheres assistidas pelos 2 blocos. Concluímos que a implementação de uma Iniciativa de Promoção, Proteção e Apoio à Amamentação na Atenção Primária à Saúde contribui para intensificar a prática do aleitamento materno exclusivo entre os lactentes menores de 6 meses.

Palavras-chave: Aleitamento materno exclusivo. Atenção básica à saúde. Efetividade. Avaliação de impacto. 
Abstract

The World Health Organization recommends exclusive breastfeeding up to 6 months of age. We conducted a study to verify the impact of an effective set of breastfeeding promotion, protection and support strategies and procedures, conducted during prenatal and pediatric care, on the prevalence of exclusive breastfeeding in the first 6 months of life. The strategies evaluated included breastfeeding support groups and home visits, while the procedures included information on the importance of early initiation, breastfeeding on demand, hazards of bottle-feeding and pacifiers to babies; guidance on positioning, attachment, breast milk expression and family planning; and providing emotional support. This set of strategies and procedures was derived from a systematic review and led to a proposal of "Ten steps to successful breastfeeding in Primary Health Care (PHC)". Based on the BabyFriendly Hospital Initiative, an evaluation method was developed and applied in the assessment of 24 PHC units located in the State of Rio de Janeiro. Thirteen units showed a fair performance, while eleven showed a poor performance. Among infants below 6 months of age, exclusive breastfeeding expectancy was 1.6 months in units with a fair performance, and 1.1 months in units with a poor performance. No significant social or demographic differences were observed between women attending both performance categories. This study led us to conclude that the implementation of a Primary Health Care Initiative to promote, protect and support breastfeeding would contribute to intensify the practice of exclusive breastfeeding among babies under 6 months of age.

Keywords: Exclusive breastfeeding. Primary health care. Effectiveness. Impact evaluation.

\section{Introdução}

O aleitamento materno exclusivo ${ }^{1}$ por 6 meses, que já vinha sendo preconizado pelo Ministério da Saúde, passou a ser recomendado pela Organização Mundial de Saúde (OMS) a partir da $54^{\text {a }}$ Assembléia Mundial de Saúde, realizada em Genebra em maio de 2001. Essa recomendação é fruto das evidências científicas acumuladas na última década ${ }^{2}$ quanto a seus benefícios para a saúde do bebê e da mãe. No entanto, ainda não existem estratégias adotadas globalmente com vistas à sua viabilização.

A Iniciativa Hospital Amigo da Criança ${ }^{3}$ (IHAC) tem contribuído, desde 1992, para o estabelecimento e incremento da amamentação. A adoção do Código Internacional de Comercialização de Substitutos do Leite Materno ${ }^{4}$ por um número crescente de países, e alguns avanços na legislação trabalhista internacional ${ }^{5}$, ocorridos recentemente, também têm contribuído para a proteção do aleitamento materno. Sabemos, porém, que a amamentação na sua forma exclusiva $^{1}$ é pouco praticada mundialmente $^{6}$. No Brasil, o tempo mediano de aleitamento materno exclusivo é de pouco mais de um mês ${ }^{7,8}$, em que pesem os avanços alcançados com o Programa Nacional de Incentivo ao Aleitamento Materno ${ }^{9}$ e medidas recentes, como o aprimoramento da Norma Brasileira de Comercialização de Alimentos para Lactentes ${ }^{10}$ em nosso país.

Muito tem sido falado sobre o papel dos grupos voluntários “mãe a mãe" de apoio à amamentação na comunidade, como as Amigas do Peito, ou La Leche League. Estes grupos, porém, são escassos, muitas vezes atingem apenas as mulheres de classe média, e tem sido pouco estudada a sua efetividade. Para explorar o potencial do nível primário de assistência no apoio à amamentação, alguns países vêm criando "passos" (ações) a serem cumpridos pelas unidades básicas de saúde, considerando que esta rede é pública e gratuita e constitui a principal responsável pelo acompanhamento das gestantes durante o pré-natal e dos bebês na puericultura ou pediatria. Es- 
sas etapas são importantes, pois é durante a gravidez que a maior parte das mulheres formula os padrões de alimentação infantil ${ }^{11,12}$, e durante os primeiros meses de vida do bebê é comum surgirem dificuldades para amamentar e pressões sociais para a introdução precoce de água, chá, leites infantis e outros $^{13,14}$.

Foi em um país da América Latina, o Chile $^{15}$, em 1995, que pela primeira vez se criou uma iniciativa para o sucesso do aleitamento materno envolvendo os consultórios que acompanham as mães. Outros países, como o Peru ${ }^{16}$, a Nicarágua ${ }^{17}$ e a Argentina ${ }^{18}$ também desenvolveram "passos" para a rede básica, sendo que na Nicarágua oito centros de saúde já foram credenciados como "Centros de Saúde Amigos". Também em países do primeiro mundo, como o Reino Unido ${ }^{19}$, em 1998, foram criados "passos" para que locais de assistência situados na comunidade fossem estimulados a apoiar, proteger e promover a amamentação.

No Rio de Janeiro, desde 1993, o planejamento e a implementação das várias ações de promoção, proteção e apoio à amamentação no Estado vêm sendo coordenados pelo Grupo Técnico Interinstitucional de Incentivo ao Aleitamento Materno ${ }^{20}$, liderado pela Secretaria de Estado de Saúde e composto por várias instituições públicas e organizações não governamentais. Seu primeiro objetivo foi a implantação da Iniciativa Hospital Amigo da Criança no Estado. Cada vez que a equipe de um hospital era treinada, a coordenação da assistência maternoinfantil da Secretaria Municipal de Saúde correspondente também era convidada para ser capacitada, a fim de que a mesma pudesse acompanhar o processo de adequação das rotinas e de treinamento dos demais profissionais de saúde. Esses coordenadores passaram a reivindicar que as equipes da rede básica fossem incluídas nos treinamentos, argumentando que não adiantava os hospitais ajudarem a mães a iniciar a amamentação se a introdução precoce de água, sucos, e mesmo fórmulas infantis muitas vezes era preconizada por profissionais da rede básica de saúde.
Passamos a investigar o que já havia sido desenvolvido nesse sentido por outros países com vistas à criação de uma proposta de atuação em aleitamento materno para a assistência primária à saúde. Foi realizada uma revisão sistemática ${ }^{21}$ para a identificação de estratégias e procedimentos conduzidos na atenção básica à saúde com efetividade na extensão da duração do aleitamento materno. Foi então estruturada uma proposta de "Dez Passos para o Sucesso da Amamentação na Atenção Básica à Saúde" e criada uma metodologia de avaliação da efetividade das unidades básicas de saúde na promoção, proteção e apoio à amamentação. Esta metodologia foi aplicada na avaliação de uma amostra proposital ${ }^{22}$ de 24 unidades básicas de saúde da Capital, Cinturão Metropolitano e Interior do Estado do Rio de Janeiro. O impacto da atuação das unidades básicas de saúde avaliadas na prevalência de aleitamento materno exclusivo é o tema deste artigo.

\section{Metodologia}

A revisão sistemática ${ }^{21}$ realizada abrangeu estudos experimentais e quasi-experimentais, publicados entre 1980 e 1999, que conduziram estratégias e procedimentos nas fases pré-natal e de acompanhamento materno-infantil, com efetividade na extensão do aleitamento materno exclusivo, completo (exclusivo ou predominante) e/ou total (exclusivo, predominante ou complementado $)^{1}$. As intervenções que se mostraram efetivas no aumento do período de amamentação exclusiva e não exclusiva foram de longa duração e combinaram procedimentos como:

- ouvir as preocupações e dificuldades das mulheres com a amamentação e proporcionar ajuda para superá-las;

- informar às gestantes sobre a importância do início precoce da amamentação;

- informar às gestantes e às mães sobre as vantagens da amamentação exclusiva e da livre demanda, sobre como o leite materno é produzido, e sobre a importância da manutenção do aleitamento materno complementado até os 2 anos ou mais; 
- alertar as gestantes e as mães sobre os riscos do uso de leites artificiais, mamadeiras e chupetas e não expô-las ao marketing destes produtos;

- orientar as gestantes e as mães quanto à pega, posição e ordenha;

- orientar as nutrizes quanto à contracepção;

As estratégias que se mostraram mais efetivas na condução destes procedimentos foram os grupos de pré-natal e de mães, e as visitas domiciliares, proporcionando apoio face a face à amamentação e envolvendo os familiares neste apoio. Em alguns estudos, sessões individuais freqüentes também se mostraram efetivas. Os procedimentos e estratégias efetivas identificados através desta revisão foram organizados em oito passos, e a infra-estrutura considerada pela IHAC enquanto indispensável para a implementação adequada das ações de aleitamento materno, em dois passos. Foi criada uma "Iniciativa Unidade Básica Amiga da Amamentação” no Estado do Rio de Janeiro, baseada nos Dez Passos para o Sucesso da Amamentação. O projeto teve o apoio do UNICEF-New York.

Partiu-se então para o desenvolvimento de um modelo de avaliação das unidades básicas de saúde nesta Iniciativa, baseado na metodologia de avaliação da $\operatorname{IHAC}^{23}$ e na trilogia Donabediana ${ }^{24}$ : estrutura-processoresultado. Para a avaliação de estrutura, foram elaborados instrumentos de observação do serviço e questionários estruturados para a entrevista de profissionais de saúde, a fim de verificar sua capacitação para a prática dos Dez Passos. Para a avaliação de processo, foram elaborados questionários estruturados para entrevistar gestantes e mães, a fim de investigar se haviam recebido as informações, orientações e apoio relativos aos passos 3 a 10. Para a avaliação de resultado, foi criado um formulário, a ser preenchido pelas auxiliares de enfermagem que pesam as crianças, sobre a forma como a clientela de bebês menores de 6 meses havia sido alimentada nas últimas 24 horas. Fatores como o trabalho materno fora do lar e o apoio recebido do hospital para ama- mentar foram também investigados através deste formulário, a fim de controlar possíveis fatores intervenientes entre a prática das unidades básicas de saúde e a prevalência de aleitamento materno exclusivo entre a clientela de bebês menores de 6 meses. Utilizou-se o software LACMAT ${ }^{25}$, desenvolvido na Argentina, para analisar os dados de alimentação infantil. Foi criado um formulário de avaliação global para a consolidação dos dados de processo e estrutura.

Esse material e a dinâmica de avaliação foram pré-testados pela primeira autora em um estudo piloto que contou com o apoio do Grupo Técnico Interinstitucional de Incentivo ao Aleitamento Materno, e envolveu cinco unidades básicas de diferentes regiões do Estado.

Todos os coordenadores municipais da assistência materno-infantil do Estado do Rio de Janeiro, que já haviam sido treinados na IHAC, foram convidados para serem treinados enquanto avaliadores da Iniciativa Unidade Básica Amiga da Amamentação. Este treinamento teve uma carga horária de 24 horas. Envolveu o debate das bases científicas dos Dez Passos para o Sucesso da Amamentação da Iniciativa Unidade Básica Amiga da Amamentação e do modelo de avaliação adotado, a aplicação prática do formulário de observação e de questionários de avaliação em um Centro de Saúde próximo, e a consolidação dos resultados. Os coordenadores materno-infantis foram orientados a selecionar uma amostra ao acaso de 10 profissionais de saúde (que já trabalhassem na unidade há mais de 4 meses) e de 10 gestantes e 10 mães de bebês menores de 1 ano (que já tivessem sido consultados 2 ou mais vezes), a serem entrevistados em diferentes dias e turnos de atendimento. Foram também capacitados a treinar os auxiliares de enfermagem na coleta de dados sobre a alimentação dos bebês menores de 6 meses e a supervisionar este processo. Os dados de alimentação infantil obtidos no estudo piloto foram transcritos para o software LACMAT $^{25} \mathrm{e}$ os avaliadores foram capacitados na sua análise. Ao final do curso, cada coordenador materno-infantil indicou en- 
tão duas a três unidades básicas de seu município, com diferentes níveis de atuação no apoio ao aleitamento materno, para serem avaliadas.

A avaliação de estrutura (passos 1 e 2) e de processo (passos 3 a 10) gerava escores globais (EG) que podiam variar de 1 (infraestrutura adequada e todos os passos cumpridos pela unidade) a 0 (infra-estrutura inadequada e nenhum procedimento realizado pela unidade). Os níveis de desempenho das unidades a serem avaliadas foram estipulados da seguinte forma: "bom" (EG de 0,67 ou acima); "regular" (EG entre 0,66 e 0,34); e "fraco" (EG de 0.33 ou abaixo).

A prevalência de aleitamento materno exclusivo entre a clientela de bebês menores de 6 meses de cada unidade e dos diferentes blocos de desempenho foi ajustada por idade pelo método de padronização direto ${ }^{26}$, em função de uma possível distribuição etária diferenciada entre elas.

\section{Resultados}

A aplicação do modelo de avaliação transcorreu de setembro de 1999 a maio de 2000 , envolvendo vinte e quatro unidades básicas de saúde do Estado, distribuídas por três áreas programáticas (AP) da Capital, quatro municípios do Cinturão Metropolitano e quatro municípios do Interior. Unidades da mesma área programática ou município eram avaliadas durante o mesmo mês. Não houve recusas entre as unidades a serem avaliadas, os profissionais de saúde ou as mulheres entrevistadas. Dos 14 coordenadores da assistência materno-infantil treinados na IHAC, 11 foram capacitados enquanto avaliadores da Iniciativa Unidade Básica Amiga da Amamentação e participaram do processo de avaliação das unidades indicadas de seus respectivos municípios. Esse processo foi conduzido em cada unidade de saúde por dois avaliadores (a primeira autora e o avaliador municipal), que aplicavam o formulário de observação e entrevistavam o diretor da unidade, profissionais de saúde do pré-natal e pediatria, gestantes e mães. As auxiliares de enfermagem aplicavam o formulário sobre alimentação infantil a todas as mães de bebês menores de 6 meses que eram pesados durante o mês de avaliação em cada unidade.

Foram entrevistadas pelos avaliadores 240 gestantes e 240 mães de bebês, e pelas auxiliares as mães de 2.458 bebês menores de 6 meses. Foram entrevistados também 24 diretores (ou chefias do setor maternoinfantil) das unidades avaliadas e 236 profissionais ou pessoal de saúde, das seguintes categorias: pediatras (25\%), obstetras (14\%), enfermeiras (15\%), auxiliares (25\%), agentes de saúde (5\%), nutricionistas (4\%) e $12 \%$ de outras categorias (incluindo clínicos gerais, assistentes sociais e psicólogos).

Foram avaliadas unidades básicas de vários tipos: centros de saúde, postos de saúde, postos de saúde da família e unidades mistas. Treze unidades avaliadas apresentaram um desempenho regular e onze um desempenho fraco (Tabela 1). Ambos os níveis de desempenho foram encontrados na amostra de unidades indicadas pelos avaliadores municipais para serem avaliadas, exceto em 3 locais: as 3 unidades de um

Tabela 1 - Tipos de unidades básicas de saúde avaliadas por categoria de desempenho Estado do Rio de Janeiro, 2000

Table 1 - Types of PHC units evaluated by performance category - State of Rio de Janeiro, 2000

\begin{tabular}{lccccc}
\hline & \multicolumn{4}{c}{ Tipos de unidades básicas de saúde avaliadas } & \\
\cline { 2 - 5 } Desempenho & $\begin{array}{c}\text { Unidade Mista } \\
\text { de Saúde }\end{array}$ & $\begin{array}{c}\text { Centro } \\
\text { de Saúde }\end{array}$ & $\begin{array}{c}\text { Posto } \\
\text { de Saúde }\end{array}$ & $\begin{array}{c}\text { Posto de Saúde } \\
\text { da Família }\end{array}$ & Total \\
\hline Regular & 1 & 6 & 4 & 2 & 13 \\
Fraco & 2 & 5 & 2 & 2 & 11 \\
Total & 3 & 11 & 6 & 4 & 24 \\
\hline
\end{tabular}


município do Interior e as 2 unidades da AP 4 da Capital foram classificadas como regulares, enquanto em um município do cinturão metropolitano ambas as unidades apresentaram um desempenho fraco. Apesar de todos os municípios não estarem igualmente representados nos 2 blocos de desempenho, as mulheres entrevistadas nestes 2 blocos apresentaram características sociodemográficas semelhantes (Tabela 2).

Mais da metade das gestantes e mães entrevistadas no bloco de unidades com melhor desempenho haviam sido informadas sobre a importância do aleitamento materno exclusivo, encorajadas a amamentar sob livre demanda, orientadas quanto aos riscos do uso da mamadeira e participado de atividades em grupo para o apoio à amamentação. Quase a metade havia sido orientada quanto à pega e posição adequadas e cerca de um terço havia sido informada sobre como o leite materno é produzido e inquirida sobre dúvidas ou dificuldades com a amamentação e ajudada a superá-las.

Todos os procedimentos investigados foram mais praticados no bloco de unidades de desempenho regular do que no bloco de desempenho fraco, exceto a observância à Norma Brasileira para Comercialização de
Alimentos para Lactentes ${ }^{27}$, que estava sendo cumprida pelas 24 unidades avaliadas: nenhuma mulher entrevistada havia sido exposta à propaganda ou recebido leites infantis, mamadeiras ou bicos na unidade.

Algumas estratégias e procedimentos eram pouco praticados, tanto nas unidades de desempenho regular quanto fraco, como a busca do envolvimento da família no apoio à amamentação (19 \% e $4 \%$, respectivamente), a orientação quanto à ordenha manual ( $11 \%$ e $4 \%$, respectivamente) e à contracepção (22\% e $14 \%$, respectivamente). A maior parte das mães entrevistadas não tinha sido informada que o uso de pílulas combinadas pode reduzir a quantidade de leite materno, nem que o método de amenorréia lactacional é uma alternativa efetiva para contracepção ${ }^{28}$. Um quarto das mães entrevistadas estava utilizando pílulas combinadas e $16 \%$ havia adotado a esterilização.

Apenas um quinto dos profissionais de saúde entrevistados havia sido treinado em cursos teórico-práticos de 18 ou mais horas sobre as vantagens e o manejo da amamentação (27\% nas unidades de desempenho regular e $11 \%$ nas de desempenho fraco). Um quarto das unidades básicas (todas classificadas como de desempenho regular)

Tabela 2 - Características sócio-demográficas das mulheres entrevistadas por categoria de desempenho - Estado do Rio de Janeiro, 2000.

Table 2 - Socio-demographic characteristics of women interviewed by performance category-State of Rio de Janeiro, 2000

\begin{tabular}{|c|c|c|}
\hline \multirow[t]{2}{*}{ Características sócio-demográficas } & \multicolumn{2}{|c|}{ №(\%) de gestantes e mães } \\
\hline & Desempenho regular & Desempenho fraco \\
\hline Idade $<20$ anos $^{*}$ & $56 / 260(21,5)$ & $47 / 220(21,4)$ \\
\hline Estudaram até a $4^{a}$ série $^{*}$ & $84 / 260(32,3)$ & $66 / 220(30,0)$ \\
\hline Não moravam com um companheiro* & $51 / 260(19,6)$ & $38 / 220(17,3)$ \\
\hline Ninguém para ajudar em casa com o bebê ${ }^{*}$ & $9 / 260(3,5)$ & $3 / 220(1,4)$ \\
\hline Eram primíparas* & $107 / 260(41,2)$ & $80 / 220(36,4)$ \\
\hline Trabalhavam fora de casa* & $51 / 260(19,6)$ & $38 / 220(17,3)$ \\
\hline Amamentaram o último bebê por menos de $6 \mathrm{~m}^{*}$ & $64 / 153(41,8)$ & $54 / 140(38,6)$ \\
\hline Receberam ajuda para amamentar do hospital ${ }^{\dagger}$ & $853 / 1387(61,5)$ & $661 / 1053(62,8)$ \\
\hline Trabalhavam fora de casa ${ }^{\dagger}$ & $210 / 1394(15,1)$ & $134 / 1053(12,7)$ \\
\hline
\end{tabular}

* Dados correspondentes às gestantes e mães de bebês $<1$ ano entrevistadas pelos 2 avaliadores

+ Dados correspondentes às mães de bebês $<6$ meses coletados pelas auxiliares na sala de preparo

"Data refer to pregnant women and mothers of $<1$ year-old babies, interviewed by two evaluators

${ }^{+}$Data refer to mothers of babies under 6 months, collected by the auxiliaries at the weighing room 
tinha normas escritas de aleitamento materno; no entanto, nenhuma cobria todos os Dez Passos da Iniciativa Unidade Básica Amiga da Amamentação.

A prevalência de aleitamento materno exclusivo encontrada na clientela de bebês menores de 6 meses acompanhados pelas unidades avaliadas foi baixa. Quase a metade $(47,9 \%)$ das mães já fornecia água, chá ou suco $(19,5 \%)$ ou outros alimentos $(28,3 \%)$ desde o primeiro mês de vida.

A prevalência da amamentação exclusiva entre os bebês menores de 6 meses foi maior no bloco de unidades de desempenho regular do que no bloco de desempenho fraco $(38,6 \%$ e $23,6 \%$, respectivamente, $p<0,001$ ), diferença esta que se apresentou consistentemente em todas as faixas etárias, e se acentuou no $5^{\circ}$ e $6^{\circ}$ meses de vida (Tabela 3). Foi realizada uma análise de sobrevivência ${ }^{29}$ com estes dados de alimentação infantil nas últimas 24 horas e encontrada uma expectativa de duração do aleitamento materno exclusivo de 1,6 meses para os bebês menores de 6 meses assistidos pelo bloco de unidades de desempenho regular, e de 1,1 meses para os bebês acompanhados pelo bloco de desempenho fraco.

Ao analisar os dois possíveis fatores de confusão controlados individualmente, observamos que o trabalho materno fora do lar pareceu exercer uma influência negativa sobre o aleitamento materno: o índice de amamentação exclusiva foi o dobro $(34,7 \%)$ entre as mães que não estavam trabalhando fora em relação às mães que trabalhavam fora (16,9\%). Já entre as mães que referiram ter recebido ajuda para amamentar da maternidade onde se deu o parto, $34 \%$ amamentava exclusivamente, contra $29 \%$ das mães que não receberam apoio do hospital. No entanto, estas duas variáveis não se comportaram como confundidoras na relação entre o desempenho dos 2 blocos de unidades e a prática de aleitamento materno exclusivo pelas mães da clientela de bebês menores de 6 meses, pois foi muito semelhante a proporção de mães dos blocos de desempenho regular e fraco que tiveram ajuda para amamentar do hospital $(61.5 \%$ e $62.8 \%$ respectivamente, $p=0,55$ ) e que trabalhavam fora $(15,1 \%$ e $12,7 \%$ respectivamente, $p=0,11$ ).

Foi verificada a associação entre a medida de resultado "prevalência de aleitamento materno exclusivo entre bebês menores de 6 meses" e a avaliação de estrutura e processo representada pelo Escore Global da unidade, sendo encontrada uma correlação moderada entre ambas (coeficiente de correlação linear de Pearson = 0,648, $p<0.01$ ), como podemos observar na Figura 1. Nas duas unidades básicas avaliadas que alcançaram os mais elevados Escores Globais (0,66 e 0,64 ) quase dois terços dos bebês menores de 6 meses estavam em aleitamento materno exclusivo (64\% e $66 \%$, respectivamente).

Tabela 3 - Aleitamento materno exclusivo por faixa etária entre a clientela de bebês menores de 6 meses por categoria de desempenho - Estado do Rio de Janeiro, 2000

Table 3 - Exclusive breastfeeding by age group for babies under 6 months of age by performance category-State of Rio de Janeiro, 2000

\begin{tabular}{|c|c|c|c|}
\hline \multirow[t]{2}{*}{ Faixa etária } & \multicolumn{2}{|c|}{$\mathrm{N}^{\circ}(\%)$ de bebês amamentados exclusivamente } & \multirow[t]{2}{*}{ pvalor } \\
\hline & Desempenho regular & Desempenho fraco & \\
\hline$<1$ mês & $167 / 274(60,9)$ & $76 / 192(39,6)$ & 0,000 \\
\hline 1 mês & $134 / 283(47,3)$ & $69 / 213(32,4)$ & 0,001 \\
\hline 2 meses & $96 / 242(39,7)$ & $56 / 199(28,1)$ & 0,015 \\
\hline 3 meses & $61 / 219(27,9)$ & $29 / 152(19,1)$ & 0,069 \\
\hline 4 meses & $54 / 214(25,2)$ & $13 / 164(7,9)$ & 0,000 \\
\hline 5 meses & $29 / 169(17,2)$ & $6 / 137(4,4)$ & 0,001 \\
\hline Total $<6 \mathrm{~m}$ & $541 / 1401(38,6)$ & $249 / 1057(23,6)$ & 0,000 \\
\hline
\end{tabular}




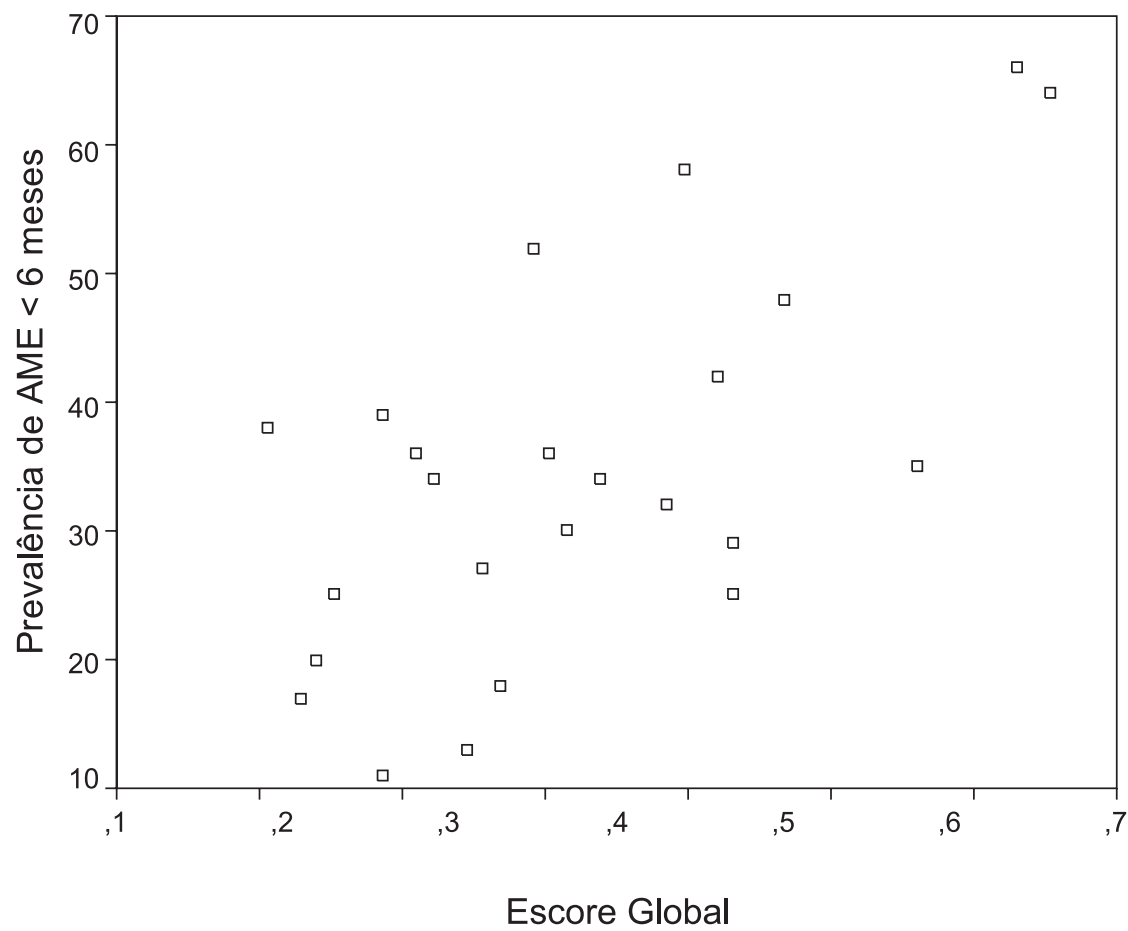

Figura 1 - Escore Global e prevalência de aleitamento materno exclusivo entre bebês menores de 6 meses nas 24 unidades básicas de saúde avaliadas - Estado do Rio de Janeiro, 2000.

Figure 1 - Global Score and prevalence of exclusive breastfeeding among babies under 6 months of age in 24 PHC units evaluated - State of Rio de Janeiro, 2000.

\section{Discussão}

A experiência de avaliação das unidades básicas de saúde foi muito rica, tanto pelo contato mais próximo com os coordenadores da assistência materno-infantil dos vários municípios que participaram do trabalho de campo, quanto pela diversidade de contextos onde se passaram as avaliações, envolvendo tanto profissionais e pessoal de saúde com níveis de capacitação e envolvimento distintos, quanto mães e gestantes com diferentes maneiras de viver, sentir e praticar a amamentação.

Foi interessante observar que o fator determinante da prevalência de aleitamento materno exclusivo encontrada nas unidades básicas de saúde avaliadas não pareceu ser o nível de complexidade destas unidades, nem o contexto rural ou urbano onde elas se inseriam. Fatores como a idade, paridade, ex- periência prévia com amamentação e escolaridade maternas apresentaram uma distribuição semelhante entre os dois blocos de unidades. A proporção de baixo peso ao nascer não foi investigada, pois nenhuma unidade era referência para bebês de risco, sendo esperada portanto uma distribuição semelhante desta variável entre as unidades avaliadas. Confundidores potenciais, como o trabalho materno e a ajuda recebida do hospital para amamentar, não pareceram influenciar a associação entre as práticas de apoio à amamentação e a duração do aleitamento materno exclusivo, por terem se distribuído de forma semelhante nos dois blocos de desempenho.

A prática de procedimentos e estratégias efetivas, identificados através da revisão sistemática ${ }^{21}$, mostrou ser o fator preponderante para a maior expectativa de duração do aleitamento materno exclusivo e para as 
maiores proporções de aleitamento materno exclusivo observadas em todas as faixas etárias, entre os bebês menores de 6 meses acompanhados pelas unidades de desempenho regular. Os índices encontrados, no entanto, ainda se situaram muito aquém dos desejados, já que mesmo nas unidades de desempenho regular quase $40 \%$ dos bebês no primeiro mês de vida já estavam recebendo outros líquidos ou alimentos, e no sexto mês de vida esta proporção ultrapassou os $80 \%$.

Foi interessante também observar que a maior efetividade encontrada no bloco de unidades de desempenho regular pareceu estar associada à prática, não de procedimentos isolados, mas de um conjunto variado de procedimentos que aliavam o apoio emocional à orientação prática no manejo da amamentação e à veiculação face a face de informações de interesse da clientela.

Foram encontradas unidades básicas de saúde de vários tipos, e nas várias regiões do Estado, fornecendo informações e orientações consistentes no manejo da amamentação, bem como apoio pessoal e em grupo para a prática de amamentar. Esta constatação parece indicar que a capacitação de profissionais e pessoal de saúde, seu crescente envolvimento enquanto equipe e o adequado gerenciamento das unidades são fatores que podem levar a um melhor aproveitamento do potencial já existente nas atuais condições da rede básica de saúde, gerando um bom desempenho das mesmas na promoção, proteção e apoio à amamentação.

O método utilizado de avaliação das práticas das unidades básicas de saúde na promoção, proteção e apoio ao aleitamento materno foi validado no trabalho de campo. Sua validade de conteúdo ${ }^{30}$ está baseada nos procedimentos e estratégias identificadas como efetivas em estender o aleitamento materno pela revisão sistemática ${ }^{21}$. Sua validade de constructo ${ }^{30}$ foi demonstrada pela associação encontrada entre a avaliação de processo e estrutura e a avaliação de resultado. Mesmo que outros fatores, não controlados, tenham contribuído em alguma medida para a prevalência de amamentação exclusiva encontrada em cada unidade, a correlação linear significativa encontrada entre o Escore Global e a prática do aleitamento materno exclusivo sugere que a aplicação em maior grau de um conjunto de estratégias e procedimentos efetivos pelas unidades avaliadas como de melhor desempenho contribuiu para a manutenção da amamentação exclusiva pelas mães de bebês menores de 6 meses.

A contribuição da rede básica de saúde para a promoção, proteção e apoio ao aleitamento materno tem sido incipiente na sua forma de organização atual, considerando o relevante papel desta categoria de serviços na atenção materno-infantil.

Considerando a evidência científica já disponível quanto a estratégias e procedimentos com efetividade na extensão do aleitamento materno exclusivo e não exclusivo, quando aplicados na assistência primária à gestante e ao binômio mãe-bebê, bem como a simplicidade e acurácia do método de avaliação desenvolvido, podemos concluir que a implementação de uma Iniciativa Global de Promoção, Proteção e Apoio à Amamentação na Atenção Primária à Saúde poderá contribuir para a extensão da amamentação exclusiva no âmbito mundial.

No entanto, ressaltamos que as características diferenciadas da rede primária de saúde existentes nas várias partes do mundo precisam ser melhor investigadas e os instrumentos de avaliação desenvolvidos necessitam ser testados em campo em outros contextos sociais e de assistência à saúde.

\section{Agradecimentos}

Os autores gostariam de agradecer à Alison E. Tedstone, da London School of Hygiene and Tropical Medicine, pela supervisão à realização da revisão sistemática. Agradecemos também à CAPES, à UNICEFNew York e à Secretaria de Estado de Saúde do Rio de Janeiro pelo apoio logístico e financeiro a este trabalho. Por fim, agradecemos à Adilcéa de Sousa, Dione Kauffman, Elda Tavares, Fernanda Sá, Giannina 
Castagnino, Luciana de Aguiar, Maria da Conceição Salomão, Mônica Carreiro, Myrian Cruz, Rosane Pereira e Tatiana Campos pela inestimável contribuição enquanto avaliadoras municipais, e à Gisele Peixoto
Barbosa, Ana Cristina dos Reis e Marcus Renato de Carvalho pela sua participação no "Curso de Avaliadores de Unidades Básicas de Saúde quanto à Promoção, Proteção e Apoio ao Aleitamento Materno".

\section{Referências}

1. World Health Organization. Indicators for assessing breast-feeding practices. Geneva; 1992.

2. World Health Organization. The optimal duration of exclusive breastfeeding: results of a WHO systematic review. Note for the press No 7. Geneva; 2 Apr 2001.

3. Saadeh R.\& Akré J. Ten steps to successful breastfeeding: a summary of the rationale and scientific evidence. Birth 1996; 23:154-60.

4. World Health Organization. The International Code of Marketing of Breast-Milk Substitutes. Geneva; 1981.

5. Rea M \& Batista LE. Proteção social à maternidade: ganhos e perdas na nova Convenção da OIT. Jornal da Rede Saúde 2000; 21: 19-20.

6. Haggerty PA \& Rutstei, SO. Demographic and Health Surveys. Comparative Studies No 30 . Breastfeeding and complementary infant feeding, and the postpartum effects of breastfeeding. Calverton, MD: Macro International Inc.; 1999.

7. BEMFAM - Sociedade Civil Bem-Estar Familiar no Brasil. Pesquisa Nacional sobre Demografia e Saúde, 1996. Rio de Janeiro: BENFAM; 1997.

8. Ministério da Saúde. Pesquisa de prevalência do aleitamento materno nas capitais e no Distrito Federal. Brasília (DF); 2001.

9. Ministério da Saúde/INAN/PNIAM. Concepção, evolução e perspectivas. Brasília (DF); 1986.

10. Ministério da Saúde. Portaria no 2051, de 8 de novembro de 2001. Norma Brasileira de Comercialização de Alimentos para Lactentes e Crianças de Primeira Infância, Bicos, Chupetas e Mamadeiras. Brasília: Diário Oficial da União - Seção 1; no 215 de 9/11/2001.

11. Neifert M, Gray J, Gary N. Factors influencing breastfeeding among adolescents. Adolesc Health Care 1988; 9: 470-3.

12. Souza IEO. O desvelar do Ser Gestante diante da possibilidade da amamentação. [tese de doutorado]. Rio de Janeiro: Escola de Enfermagem Ana Nery da UFRJ; 1993.

13. Huffman SL. Determinants of breastfeeding in developing countries: overview and policy implications. Stud Fam Plan 1984; 15(4): 170-83.
14. Bryant CA. The impact of kin, friend and neighbor networks on infant feeding practices. Soc Sci Med 1982; 16: 1757-65.

15. Ministerio de Salud de la Republica de Chile, Comisión Nacional de Lactancia Materna. Criterios de evaluacion de consultorios que apoyan la iniciativa conjunta OMS-UNICEF Hospitales Amigos. Santiago; 1995.

16. Giga VTS, Sanez NC. Centros y Puestos promotores de la alimentación infantil: Once pasos hacia una adecuada alimentación infantil. CEPREN; 1996.

17. Ministry of Education of Nicaragua et al. Eleven Steps to PHC for successful breastfeeding. In: BabyFriendly Hospital Initiative - Case studies and progress report. New York: UNICEF Programme Division; 1999.

18. Vallone F, Mercer R. Programa Materno Infantil, Ministerio de Salud. 10 acciones del primer nivel de atención en apoyo de la lactancia materna. Buenos Aires; 1996.

19. UNICEF UK Baby Friendly Initiative. A Seven Point Plan for the protection, promotion and support of breastfeeding in community health care settings. London. UNICEF UK BFI; 1998.

20. Secretaria de Estado de Saúde. Resolução no 837, de 30 de março de 1993. Compõe o Grupo Técnico Interinstitucional de Incentivo ao Aleitamento Materno do Estado do Rio de Janeiro. Diário Oficial do Estado do Rio de Janeiro - Ano XIX; nº 66; Parte 1; 12/04/1993.

21. de Oliveira MIC, Camacho LAB, Tedstone AE. Extending breastfeeding duration through primary care: a systematic review of prenatal and postnatal interventions. J Hum Lact 2001; 17(4): 326-43.

22. Worthen BR, Sanders JR, Fitzpatrick JL. Program Evaluation. Alternative Approaches and Practical Guidelines. Second Edition. New York: Longman; 1997.

23. United Nations Children's Fund, World Health Organization, Wellstart International. Baby-Friendly Hospital Initiative: external assessors' manual. WHO/ UNICEF; 1992

24. Donabedian A. Explorations in quality assessment and monitoring. Vol I: The definition of quality and approaches to its assessment. Michigan. Health Administration Press; 1980. 
25. Vallone F, Jaquenod M. LACMAT 3.4 (Software). Buenos Aires; 1998.

26. Rothman KJ. Modern Epidemiology. Boston: Little, Brown and Company; 1986.

27. Ministério da Saúde. Resolução no 31, de 12 de outubro de 1992. Norma Brasileira para Comercialização de Alimentos para Lactentes. Brasília (DF): Conselho Nacional de Saúde; 1993.
28. Family Health International. Consensus statement: breastfeeding as a family planning method. Lancet 1988; ii, 1204-5.

29. Kleinbaum DG. Survival analysis: a self-learning text Statistics in Health Sciences. New York: Springer; 1997.

30. Carmines EG \& Zeller RA. Reliability and validity assessment. Beverly Hills. London: SAGE Publications; 1979.

Recebido em 04/01/02; aprovado em 15/08/02 